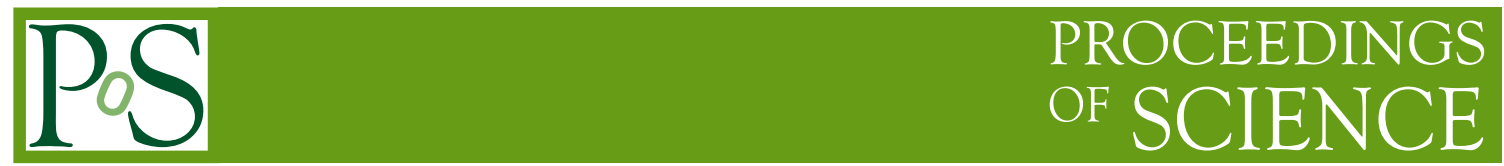

\title{
Dark photon searches with the ATLAS detector at the LHC
}

\author{
Hassnae EI Jarrari ${ }^{a, *}$, on behalf of the ATLAS Collaboration \\ ${ }^{a}$ Mohammed V University in Rabat \\ E-mail: hassnae.el.jarrari@cern.ch
}

\begin{abstract}
Many extensions to the Standard Model (SM) introduce a hidden or dark sector (DS) to provide candidates for dark matter in the universe and an explanation to astrophysical observations such as the positron excess observed in the cosmic radiation flux. This hidden sector could rise from an additional $U(1)_{d}$ gauge symmetry. ATLAS has searched for the gauge boson of the DS, which could be a massless or massive dark photon that either kinetically mixes with the SM photon or couples to the Higgs sector via some mediators. If dark photons decay in turn to SM particles with a significant branching ratio, we could either observe measurable deviations in some particular Higgs boson decay channels or new exotic signatures that would be accessible at the Large Hadron Collider (LHC) energies.
\end{abstract}

The Eighth Annual Conference on Large Hadron Collider Physics-LHCP2020

25-30 May, 2020

online

*Speaker 


\section{Introduction}

Several models propose exotic Higgs boson decays as evidence of new physics. Potential decay modes are essential to study the dark sector and its components. This proceeding summarizes two searches for dark photons based on data collected by ATLAS [1] at a $13 \mathrm{TeV}$ center-of-mass energy during the 2015 and 2016 periods of the LHC [2] Run 2, corresponding to a luminosity of $36 \mathrm{fb}^{-1}$.

\section{Models for dark photon searches}

Dark sectors are scenarios in which the new physics structure appears somehow disentangled from the SM. In such scenarios, portals generated by quantum mechanics propagate interactions between dark and SM sectors. ATLAS has performed the following searches:

- Scalar portal $(\mathrm{H})$ : Add a dark singlet $(\phi / S)$ that spontaneously breaks $U(1)$ and mixes with the SM Higgs boson (through the Higgs mixing parameter $k$ ).

$$
\mathrm{V}_{0}(H, S)=-\mu^{2}|H|^{2}+\lambda|H|^{4}-\mu_{S}^{2}|S|^{2}+\lambda_{S}|S|^{4}+k|S|^{2}|H|^{2} .
$$

- Vector portal (A): Add a $U(1)^{\prime}$ group whose massive "dark" gauge boson $\left(A^{\prime} / Z_{d} / \gamma_{d}\right)$ mixes kinetically with the SM photon (through the kinetic mixing parameter $\epsilon$ ).

$$
\mathrm{L} \supset-\frac{1}{4} \hat{B}_{\mu \nu} \hat{B}^{\mu \nu}-\frac{1}{4} \hat{Z}_{D \mu \nu} \hat{Z}_{D}^{\mu v}+\frac{1}{2} \frac{\epsilon}{\cos \theta} \hat{Z}_{D \mu \nu} \hat{B}^{\mu \nu}+\frac{1}{2} m_{D, 0}^{2} \hat{Z}_{D}^{\mu} \hat{Z}_{D \mu}
$$

\section{Dark photon searches with the ATLAS detector}

ATLAS has searched for low mass $\left(\gamma_{d}\right)$ and high mass $\left(Z_{d}\right)$ dark photons from different physics processes with masses up to $60 \mathrm{GeV}$. Two main categories have been considered in this proceeding:

\subsection{Higgs boson decays to beyond-the-SM light bosons in four-lepton events}

Existing measurements constrain the non-SM branching ratio of the Higgs boson to less than $30 \%$ at $95 \%$ confidence level (CL). Due to the extremely narrow decay width of the SM Higgs boson and given the possible "portal" it provides, the addition of even a small coupling to a new light state could open up sizeable new decay modes. This search considers a Higgs boson $\left(m_{H}=125 \mathrm{GeV}\right)$ decays to four leptons $(l=e$ or $\mu)$ via an intermediate state which contains one or two on-shell, promptly decaying bosons: $H \rightarrow Z X / X X \rightarrow 4 l$, where $X$ is a new vector boson $Z_{d}$ (Figure 1) or pseudoscalar $a$ with mass between 1 and $60 \mathrm{GeV}$ [3].

The search for $H \rightarrow Z X \rightarrow 4 l$ is performed in an $X$ mass range between $15 \mathrm{GeV}$ and $55 \mathrm{GeV}$, while the $H \rightarrow X X \rightarrow 4 l$ search is done for $15 \mathrm{GeV}<m_{X}<60 \mathrm{GeV}$ considering $4 e, 2 e 2 \mu$, and $4 \mu$ final states and for $1 \mathrm{GeV}<m_{X}<15 \mathrm{GeV}$ considering only the $4 \mu$ final state, respectively.

The main backgrounds of this study are estimated from simulation of $H \rightarrow Z Z^{*} \rightarrow 4 l$ and di-boson production via $Z Z^{*} \rightarrow 4 l$. Figure 2 shows two- and four-lepton mass distributions for the data, the SM backgrounds and three signal points for the $H \rightarrow Z Z_{d} \rightarrow 4 l$ model where the signal strength corresponds to a branching ratio $\beta\left(H \rightarrow Z Z_{d} \rightarrow 4 l\right)=\frac{1}{3} \beta\left(H \rightarrow Z Z^{*} \rightarrow 4 l\right)$. Figure 2 also shows the distributions from the $H \rightarrow X X \rightarrow 4 l$ signal corresponding to an expected yield normalized to $\sigma\left(p p \rightarrow H \rightarrow Z_{d} Z_{d} \rightarrow 4 l\right)=\frac{1}{10} \sigma_{S M}\left(p p \rightarrow H \rightarrow Z Z^{*} \rightarrow 4 l\right)$. Figure 3 shows 95\% CL upper bounds on the effective Higgs mixing parameter $k^{\prime}=k \times m_{H}^{2} /\left|m_{H}^{2}-m_{S}^{2}\right|$ and on $\epsilon$. 

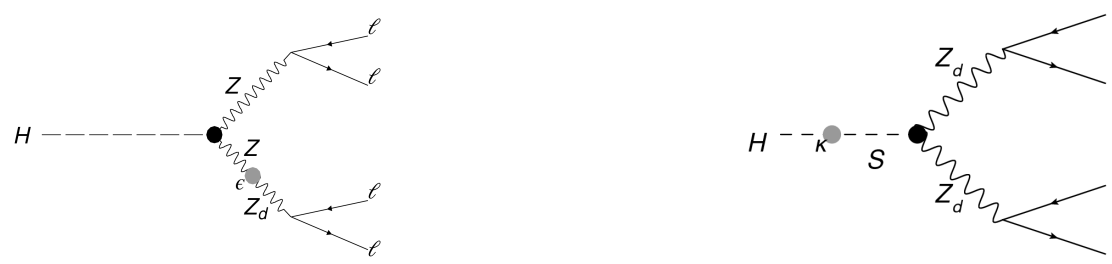

Figure 1: Exotic Higgs boson decays to four leptons induced by intermediate dark vector bosons via (left) the hypercharge portal through $\epsilon$ and (right) the Higgs portal through $k$, where $S$ is a dark Higgs boson [3].
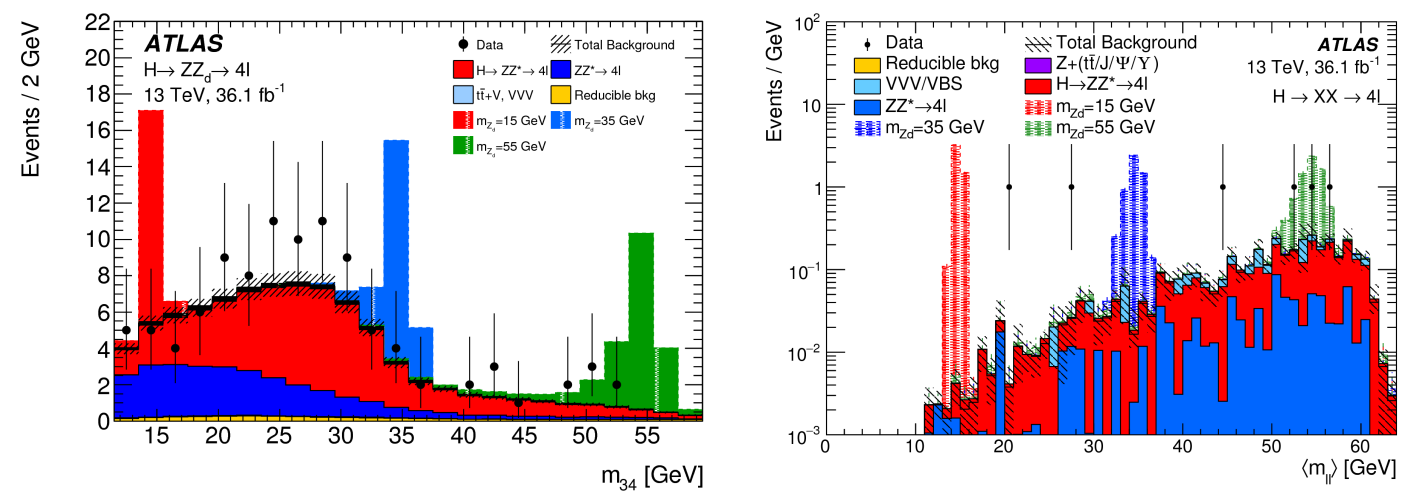

Figure 2: Left: distribution of $m_{34}$ for data and background events in the mass range $m_{4 l} \in[115,130] \mathrm{GeV}$ after the $H \rightarrow Z X \rightarrow 4 l$ selection. Right: distribution of $<m_{l l}>=\frac{1}{2}\left(m_{12}+m_{34}\right)$ for events selected in the $H \rightarrow X X \rightarrow 4 l$ analysis for $15 \mathrm{GeV}<m_{X}<60 \mathrm{GeV}$ [3].
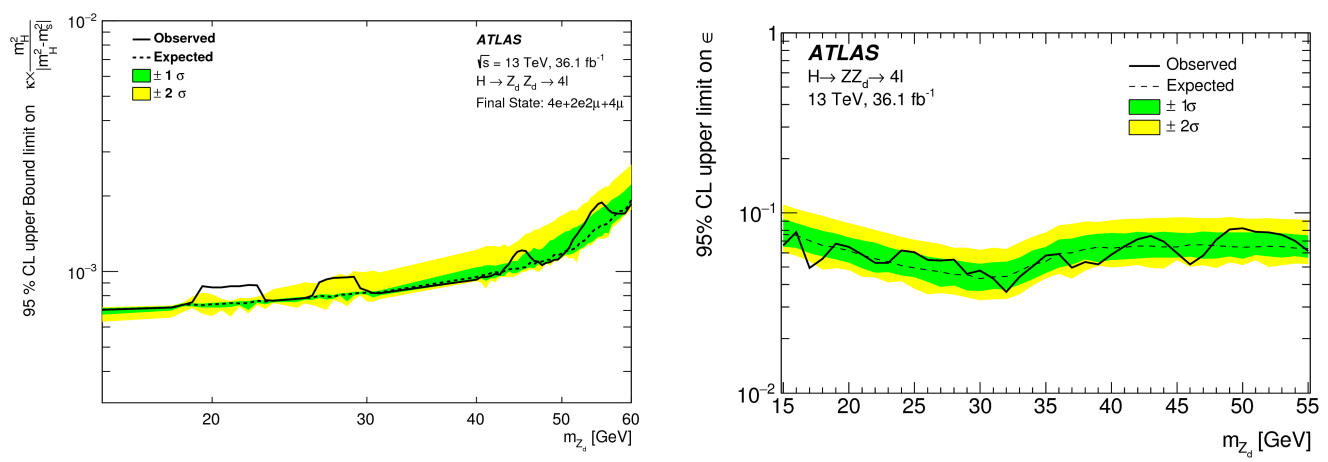

Figure 3: $95 \%$ CL upper bounds on $k^{\prime}$ and $\epsilon$ as a function of $m_{Z_{d}}$ [3].

\subsection{Light long-lived neutral particles decaying into collimated leptons or light hadrons}

This search investigates the case where DS and SM couple via a vector portal, in which a $\gamma_{d}$ mixes kinetically with the SM photon and decays into SM leptons and light quarks [4]. The lifetime of the $\gamma_{d}$ is determined through the kinetic mixing term $(\epsilon)$ which can vary between $10^{-11}$ and $10^{-2}$. For a small $\epsilon$ value, the $\gamma_{d}$ has a long lifetime, so that it decays at a macroscopic distance from its production point. The FRVZ model [5, 6] is the benchmark of this search. Figure 4 shows the two processes considered, where the dark photons are expected to be produced with large boosts due to their small mass, resulting in collimated groups of leptons $(\mu)$ or light hadrons $(h)$ in a jet-like structure, referred to hereafter as dark-photon jets (DPJs). Two main channels are considered: the $\mu \mathrm{DPJ}-\mu \mathrm{DPJ}$ channel, with cosmic rays muons as the main background, and the $h \mathrm{DPJ}-h \mathrm{DPJ}$ 


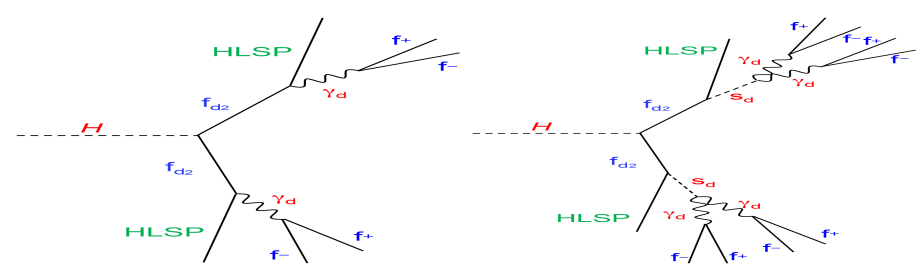

Figure 4: Dark fermion $f_{d 2}$ decays into a hidden lightest stable particle (HLSP) and a $\gamma_{d}$ (left) or a dark scalar $s_{d}$ that in turn decays into a pair of dark photons (right). Then $\gamma_{d}$ decays into SM fermions [4].
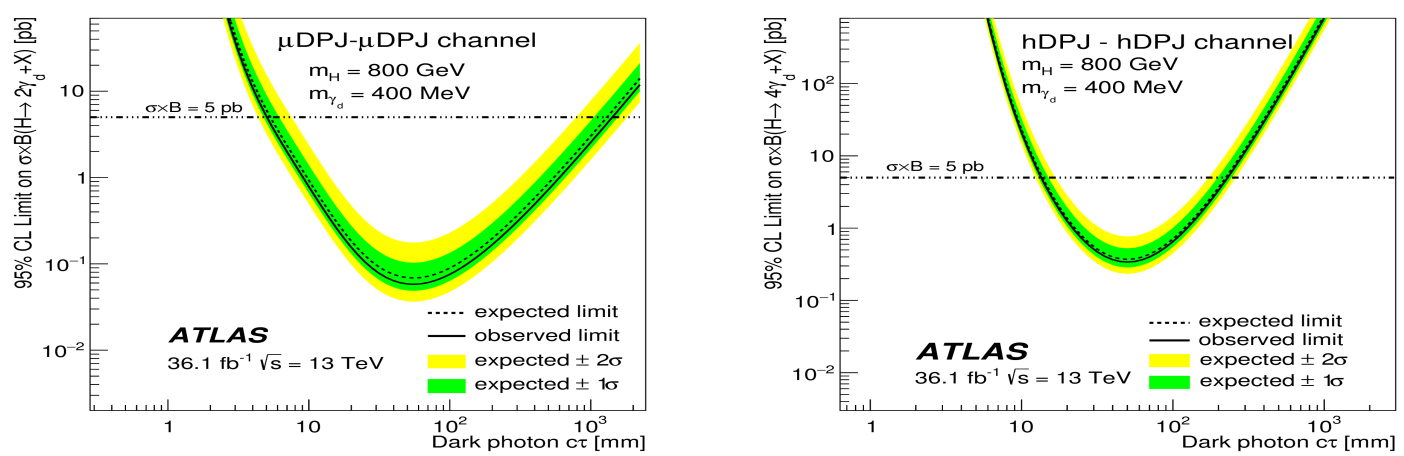

Figure 5: 95\% CL upper limits on $\sigma \times B$ for the processes $H \rightarrow 2 \gamma_{d}+X$ (left) and $H \rightarrow 4 \gamma_{d}+X$ (right) [4].

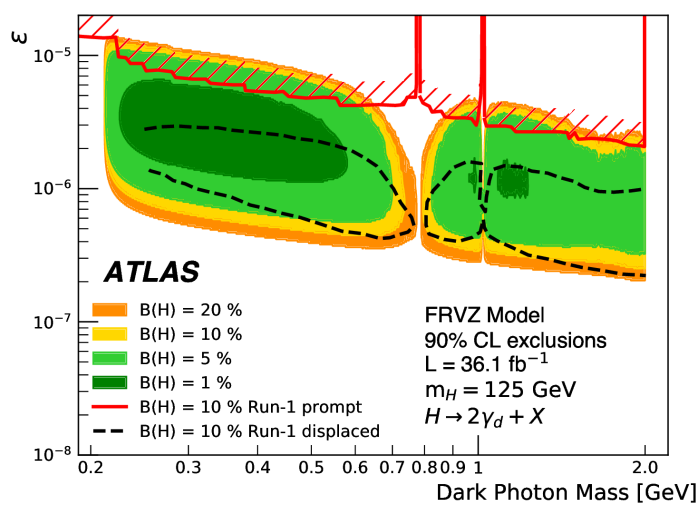

Figure 6: Run 1 and partial Run 2 combined results assuming branching ratios from 1\% to 20\% [4].

channel, where multi-jet production is the main background. Figure 5 shows upper limits at $95 \%$ CL on the cross-section times branching fraction in the two channels. This search results in an exclusion region at $90 \% \mathrm{CL}$ for $H \rightarrow 2 \gamma_{d}+X$ as a function of the $\gamma_{d}$ mass and $\epsilon$ (Figure 6).

\section{Conclusion}

The dark photon searches with the ATLAS detector at the LHC resulted in no significant excess of events above SM background predictions. Upper limits at 95\% CL are set on model-independent fiducial cross-sections and on the Higgs boson decay branching ratios to vector and pseudoscalar bosons in two benchmark models. Limits on the production cross-section times branching fraction as a function of the proper decay length of $\gamma_{d}$ are reported. 


\section{References}

[1] ATLAS Collaboration, JINST 3 (2008) S08003

[2] L. Evans and P. Bryant (editors), JINST 3 (2008) S08001

[3] ATLAS Collaboration, JHEP 06 (2018) 166, arXiv:1802.03388v2 [hep-ex]

[4] ATLAS Collaboration, Eur. Phys. J. C 79 (2019) 481, arXiv:1909.01246v2 [hep-ex]

[5] A. Falkowski, J. T. Ruderman, T. Volansky and J. Zupan, JHEP 05 (2010) 077, arXiv:1002.2952v2 [hep-ph]

[6] A. Falkowski, J. T. Ruderman, T. Volansky and J. Zupan, Phys. Rev. Lett. 105 (2010) 241801, arXiv:1007.3496v1 [hep-ph] 